\title{
Vorschau 6|2009
}

\section{Inbound Marketing}

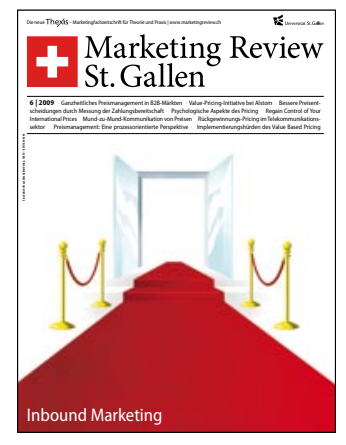

Ergreifen Kunden die Initiative beim Kauf, so stimmt für sie die Sache, der Zeitpunkt und der Verkaufskanal. Kampagnen und Aktionen von Unternehmen treffen aber immer weniger. Inbound Marketing fördert und nutzt die Initiativen der Kunden. Gelingt es, kompetent und flexibel auf deren Anliegen einzugehen, so ist das ein Volltreffer. Gleichzeitig geht modernes Marketing (von Web 2.0 über Communities bis hin zu Kundenintegration) generell davon aus, dass Kunden aktiver werden und mehr beeinflussen.

Die sechste Ausgabe der Marketing Review St. Gallen 2009 widmet sich dem Thema "Inbound Marketing". Autoren aus Wissenschaft und Praxis entwickeln bestehende Ansätze von Beschwerdemanagement bis Kundenkontaktzentren weiter, identifizieren neue Ansätze für das Management von aktiven Kunden und zeigen das Potenzial im Kundendialog. Ebenso wird untersucht, wie aktive Kunden gemeinsam mit Unternehmen Werte schöpfen.

\section{Impressum}

\author{
Marketing Review St. Gallen \\ Die neue Thexis - \\ Fachzeitschrift für Marketing \\ 26. Jahrgang \\ www.marketingreview.ch
}

Verlag

Gabler Verlag/GWV Fachverlage GmbH

Abraham-Lincoln-Straße 46

65189 Wiesbaden

Postfach 1546, 65173 Wiesbaden

Geschäftsführer

Dr. Ralf Birkelbach

Albrecht F. Schirmacher

Gesamtleitung Anzeigen

Thomas Werner

Gesamtleitung Produktion

Christian Staral

Gesamtleitung Vertrieb

Gabriel Göttlinger

Verlagsbereichsleitung

Maria Akhavan

\section{Herausgeber}

Prof. Dr. Christian Belz

Prof. Dr. Thomas Bieger

Prof. Dr. Andreas Herrmann

Prof. Dr. Sven Reinecke

Prof. Dr. Thomas Rudolph

Prof. Dr. Marcus Schögel

Prof. Dr. Torsten Tomczak

\section{Redaktionsleitung}

Universität St. Gallen

Dipl.-Kfm. Johannes Hattula

Tel.: +41 (0)71 2242876

johannes.hattula@unisg.ch

Verlagsmitarbeiterin: Karin Halder
Redaktionsleitung Gabler Verlag

Barbara Roscher

Tel.: +49 (0)611 7878-233

barbara.roscher@gabler.de

\section{Redaktion}

Volker Haßmann

Tel.: +49 (0)6131 6030485

vhassmann@t-online.de

Abonnentenbetreuung

VVA-Zeitschriften-Service

Postfach 777, 33310 Gütersloh

Tel.: +49 (0)5241 8019-68

Fax: +49 (0)5241 8096-20

Produktmanagement

Jens Fischer

Tel.: +49 (0)611 7878-340

jens.fischer@gwv-fachverlage.de

\section{Key Account Management}

Sabine Schüler

Tel.: +49 (0)611 7878-178

Fax: +49 (0)611 7878-140

sabine.schueler@gwv-media.de

Es gilt die Anzeigenpreisliste Nr. 2 vom 01.01.2009

\section{Anzeigendisposition}

Barbara Gerlach,

Tel.: +49 (0)611 7878-198

Fax: +49 (0)611 7878-443

barbara.gerlach@gwv-fachverlage.de

Layout und Produktion

Markus Paulus

Tel.: +49 (0)611 7878-171

markus.paulus@springer.com

Titelbild

Gunter Rubin

rubin@reizend.com
Bezugsmöglichkeit

Das Heft erscheint sechsmal jährlich. Bezugspreise Print + Online (inkl. MwSt. und Versand): Deutschland: 155,- EUR,

für Studenten 93,- EUR

Schweiz: ca. 263,- CHF

Weltweit: 177,- EUR,

für Studenten 103,- EUR

Einzelheftpreis: 34,- EUR zzgl. Versand

Bezugspreise E-Paper/Online only (inkl. MwSt):

Weltweit: 133,- EUR, ca. 226,50 CHF, für Studenten 80,- EUR, ca. 136,- CHF

Das Abonnenment kann jederzeit zur nächsten erreichbaren Ausgabe schriftlich mit Nennung der Kundennummer gekündigt werden. Eine schriftliche Bestätigung erfolgt nicht. Zuviel gezahlte Beträge für nicht gelieferte Ausgaben werden zurückerstattet.

\section{Druck und Verarbeitung}

Stürtz, Würzburg

Die Zeitschrift und alle in ihr enthaltenen einzelnen Beiträge und Abbildungen sind urheberrechtlich geschützt. Jede Verwertung außerhalb der engen Grenzen des Urheberrechtes ist ohne Zustimmung des Verlages unzulässig und strafbar. Das gilt insbesondere für Vervielfältigungen, Übersetzungen, Mikroverfilmungen und die Einspeicherung in elektronischen Systemen. Nachdruckgenehmigung kann die Redaktion erteilen. Für unverlangt eingesandte Beiträge und Rezensionsexemplare wird nicht gehaftet. Jede im Bereich eines gewerblichen Unternehmens hergestellte oder benützte Kopie dient gewerblichen
Zwecken gem. §-54-(2)-UrhG und verpflichtet zur Gebührenzahlung an die VG WORT, Abteilung Wissenschaft, Goethestr. 49, 80336 München, von der die einzelnen Zahlungsmodalitäten zu erfragen sind.

Alle Rechte vorbehalten. Kein Teil dieser Zeitschrift darf ohne schriftliche Genehmigung des Verlages vervielfältigt oder verbreitet werden. Unter dieses Verbot fällt insbesondere die gewerblicheVervielfältigung per Kopie, die Aufnahme in elektronische Datenbanken und die Vervielfältigung auf CD-Rom und allen anderen elektronischen Datenträgern.

\section{Hinweise für Autoren}

Der Autor ist mit derVeröffentlichung seines Beitrags damit einverstanden, dass sein Beitrag außer in der Zeitschrift auch durch Lizenzvergabe in anderen Zeitschriften (auch übersetzt), durch Nachdruck in Sammelbänden (z. B. zu Jubiläen der Zeitschrift oder des Verlages oder in Themenbänden), durch längere Auszüge in Büchern des Verlages auch zu Werbezwecken, durch Vervielfältigung und Verbreitung aufCD-ROM oder anderen Datenträgern, durch Speicherung auf Datenbanken, deren Weitergabe und den Abruf von solchen Datenbanken während der Dauer des Urheberrechtsschutzes an dem Beitrag im In- und Ausland vom Verlag und seinen Lizenznehmern genutzt wird.

(c) Gabler Verlag/GWV Fachverlage $\mathrm{GmbH}$, Wiesbaden 2009.

Der Verlag ist ein Unternehmen von Springer Science+Business Media.

ISSN 1865-6544 